\title{
The Circumgalactic Medium of Andromeda
}

\author{
Nicolas Lehner ${ }^{1}$ and the Project AMIGA team \\ ${ }^{1}$ Department of Physics, University of Notre Dame, 225 Nieuwland Science Hall, Notre Dame, \\ IN 46556 \\ email: n.lehner@nd.edu
}

\begin{abstract}
Our view of galaxies has been transformed in recent years with diffuse halo gas surrounding galaxies that contains at least as many metals and baryons as their disks. While single sight lines through galaxy halos seen in absorption have provided key new constraints, they provide only average properties. Our massive neighbor, the Andromeda (M31) galaxy, provides an unique way to study its circumgalactic medium whereby we can study it using not one or two, but $\sim 36$ sightlines thanks to its proximity. With our Large HST program - Project AMIGA (Absorption Maps In the Gas of Andromeda), our goals are to determine the spatial distribution of the halo properties of a $L *$ galaxy using 36 background targets at different radii and azimuths. In this brief paper, I discuss briefly the scientific rationale of Project AMIGA and some early science results. In particular, for the first time we have demonstrated that M31 has a gaseous halo that extends to $R_{\mathrm{vir}}$ with as much as metal and baryonic masses than in its disk and has substantial change in its ionization properties with more highly ionized gas found at $R \sim R_{\mathrm{vir}}$ than cooler gas found near the disk.
\end{abstract}

Keywords. galaxies: halos — galaxies: individual (M31) — Local Group — quasars: absorption lines

\section{Introduction}

The means by which galaxies acquire, consume, enrich, and recycle their gas encompass some of the most important processes in astrophysics. These processes occur in the circumgalactic medium (CGM). Many empirical results indicate that the CGM plays a major role in galaxy evolution and is a massive component of galaxies, including a CGM mass approximately equal to the stellar mass for $\sim L *$ galaxies (e.g., Tumlinson et et al. 2011; Stocke et al. 2013 Werk et et al. 2014). However, a significant limitation in interpreting these empirical results in the context of new high-resolution simulations is that only average properties of the CGM are robustly derived from traditional QSO absorption-line techniques for examining halo gas. In the rare cases where there is a UVbright QSO behind a galaxy, the CGM is probed along a single "core sample" through the halo of each galaxy. These measurements are then aggregated into a statistical map, where galaxies with different inclinations, sizes, and environments are blended together and the radial-azimuthal dependence of the CGM is essentially lost. All sorts of biases can result: phenomena that occur strongly in only a subset of galaxies can be misinterpreted as being weaker but more common, and genuine trends with mass or star formation rate can be misinterpreted as simply scatter with no real physical meaning. This is a major hindrance to rigorous estimates of the CGM mass, densities, kinematics, and metallicities. Overcoming this limitation is particularly important since cosmological simulations show a strong geometrical structure in the CGM that is directly related to the nature of flows in and out of galaxies that regulate their evolution (Stewart et al. 2011; Faucher-Giguère et al. 2015). 


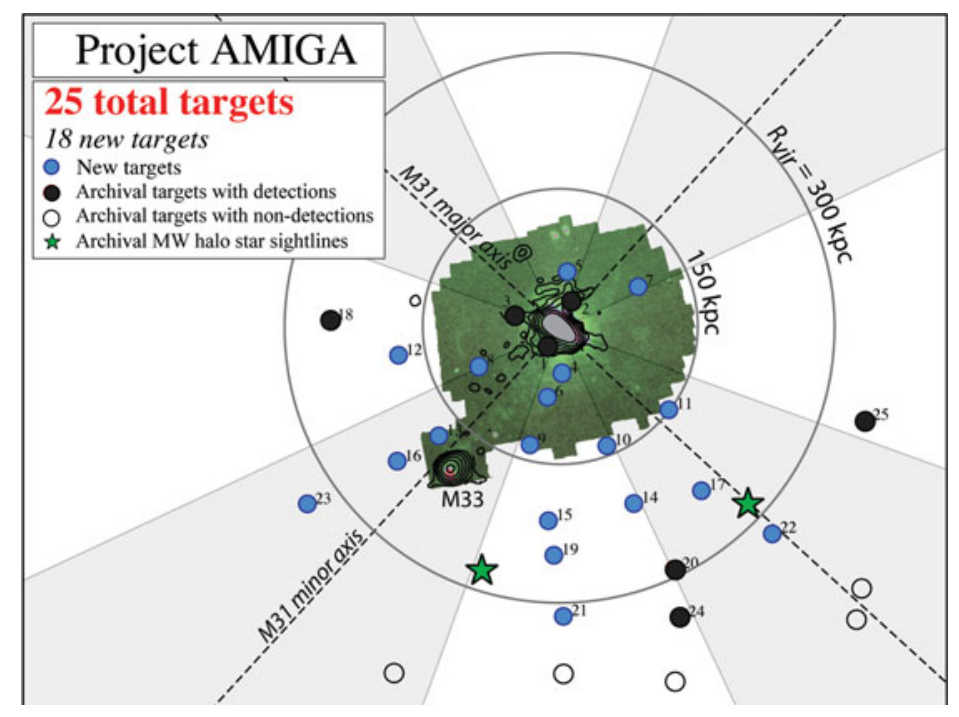

Figure 1. Distribution of the targets in our sample within $\sim 1.5 R_{\mathrm{vir}}$ from M31 (overplotted in green is the footprint of the The Pan-Andromeda Archaeological Survey, McConnachie et al. 2009). The red circles and open circles are detection in and absence of absorption from the M31 CGM gas based on the data assembled by Lehner et et al. (2015). The approved targets from our approved HST Cycle 23 program are in blue and about half of the targets in the sample still need to be observed. Beyond $1.1 R_{\mathrm{vir}}$, there is little evidence for any M31 CGM gas. When Project AMIGA is complete, we will be able for the first time study the spatial variation at various impact parameters $(R)$ and azimuths $(\Phi)$ (along the projected minor and major axes and in-between).

\section{Project AMIGA}

Thus the present state of affairs is that state-of-the-art simulations that best capture the true physics of feedback are constrained by only the average properties of the galaxies and CGM they are intended to represent. Understanding how baryons flow through the CGM of galaxies requires a global kinematic and surface density map of the gas in low and high ionization states to interpret the interplay of expelled and accreted gas. In the HST Cycle 23, our Large program Project AMIGA was approved to overcome these current limitations. With Project AMIGA, we "zoom in" on the ideal well-resolved galaxy Andromeda - using the only instrument that can do it: HST/COS. Our sample will consist of 25 sightlines (18 of them are being observed as part of our HST Cycle 23 program) probing the CGM within $\sim 1.1 R_{\mathrm{vir}}$ from M31 $\left(1 R_{\mathrm{vir}}=300 \mathrm{kpc}\right.$ for M31) and 11 beyond $\sim 1.1 R_{\text {vir }}$ from M31 (see Fig. 1). These observations will allow us for the first time to map the distribution of metals and gas flows in the CGM of a single galaxy, sampling the CGM well as a function of radial distance $(R)$ and azimuth $(\Phi)$ out to the virial radius of M31. Project AMIGA will overcome the major limitation of current observations by resolving the interplay of accretion and feedback within the CGM, constraining the degree of physical structure within a single galaxy halo, and providing a testbed for new zoom-simulations of galaxies.

\section{Results from the pilot study: Discovery of the CGM of M31}

In our pilot study to determine the feasibility of Project AMIGA (Lehner et et al. 2015 for all the details), we mined the HST/COS archive for sightlines piercing the M31 halo within a projected distance $2 R_{\mathrm{vir}}$ and observed with the $\mathrm{G} 130 \mathrm{M} / \mathrm{G} 160 \mathrm{M}$ gratings. We 


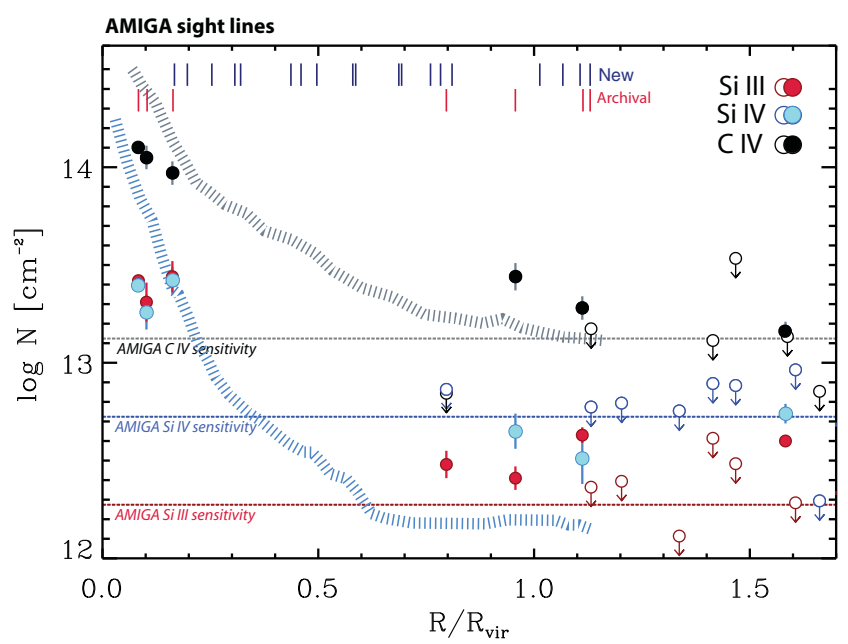

Figure 2. Results from our pilot study (Lehner et et al. 2015): The column densities of SiIII, SiIV, CIV of the M31 CGM gas at $-310 \leqslant v_{L S R} \leqslant-150 \mathrm{~km} \mathrm{~s}^{-1}$ are shown against the projected distance from M31. Open circles with downward arrows are non-detections of M31 CGM gas, mostly beyond $1.1 R_{\mathrm{vir}}$. The horizontal lines show our sensitivity limit for these key ions. The thick dashed lines show models from Ford et et al. (2014). These models appear to be appropriate for CIV, but not for SiIV (which behaves like the low ions in these models), possibly because they are only averaged over many galaxies. With the complete sample, we will be able to explore the changes in ionization with $R$ and $\Phi$ in much greater details.

found 18 sightlines, but only 7 at $R \leqslant R_{\text {vir }}$ (Fig. 1). Even this small sample shows striking results: M31 has an extended CGM out to about $1.1 R_{\mathrm{vir}}$, while the covering fraction plummets to near zero $(1 / 11)$ between $1.1 R_{\mathrm{vir}}$ and $2 R_{\mathrm{vir}}$ (Figs. 1 and 2 ). This finding echoes the "statistical" maps of other low- $z$ studies which show that CGM becomes weak and metals rare beyond $R_{\mathrm{vir}}$, so that physical diagnostics of density and metallicity are no longer readily available (e.g., Werk et al. 2014). There is also a drastic change in the ionization properties, as the gas is more highly ionized at $R \sim R_{\mathrm{vir}}$ than at $R<0.2 R_{\mathrm{vir}}$ (a trend much less ambiguous in a single galaxy than it would be in a sample of varying mass probed at varying fractions of $R_{\mathrm{vir}}$ ). Finally, these few measurements already imply that M31 CGM metal and baryonic masses within $R_{\mathrm{vir}}$ could be comparable to the respective masses in the M31 disk (as found for $z \sim 0.2 L *$ galaxies; see, e.g., Peeples et al. 2014; Werk et al. 2014). However, the mass is uncertain because the metal surface densities $(N)$ and covering fractions $\left(f_{c}\right)$ with $R$ and $\Phi$ are poorly constrained from the 7 existing sight lines with $R_{\mathrm{vir}}$, but this will greatly improve once the entire data from Project AMIGA have been obtained.

\section{References}

Faucher-Giguère, C.-A., et al. 2015, MNRAS, 449, 987.

Ford, A. B., et al. 2014, MNRAS, 444, 1260.

Lehner, N., Howk, J. C., \& Wakker, B. P. 2015, ApJ, 804, 79

McConnachie, A. W., et al. 2009, Nature, 461, 66.

Peeples, M. S., et al. 2014, ApJ, 786, 54.

Stewart, K. R., et al. 2011, ApJ, 735, L1.

Stocke, J. T., et al. 2013, ApJ, 763, 148.

Tumlinson, J., et al. 2011, Science, 334, 948.

Werk, J. K., et al. 2014, ApJ, 792, 8 